\title{
APUNTES PARA UN ANÁLISIS DE LA MULTICULTURALIDAD EN EL MERCOSUR (ARGENTINA Y BRASIL)
}

\author{
APONTAMENTOS PARA UMA ANÁLISE DA MULTICULTURALIDADE NO \\ MERCOSUL (ARGETINA E BRASIL)
SUGGESTIONS FOR AN ANLYSIS OF MULTICULTURALISM IN THE MERCOSUR (ARGENTINA AND BRAZIL)

\section{PISTES POUR UNE ANALYSE DU MULTICULTURALISME AU SEIN DU MERCOSUR (ARGENTINE ET BRÉSIL)}

DOI: 10.5533/1984-2503-20124205

\section{Fernando Milano ${ }^{1}$}

\section{RESUMEN}

El objeto del presente trabajo es brindar algunas ideas acerca de las culturas jurídicas comparadas en el ámbito del Mercosur, con especial referencia a la Argentina y al Brasil. Palabras-clave: Multiculturalidad; Mercosur; Brasil y Argentina.

\section{RESUMO}

O objetivo do presente estudo é desenvolver algumas ideias acerca das culturas jurídicas comparadas no âmbito do Mercosul, com referência especial a Argentina e ao Brasil. Palavras-chave: Multiculturalidade; Mercosul; Brasil e Argentina.

\section{ABSTRACT}

The object of the present study is to develop thoughts on legal cultures within countries forming the Mercosur, with special regard to Argentina and Brazil.

Key-words: Multiculturalism; Mercosur; Brazil; Argentina.

\footnotetext{
${ }^{1}$ Profesor Regular Adjunto de Derecho de la Integración de la Facultad de Derecho de la Universidad de Buenos Aires. Profesor Adjunto de Sistemas Legales Contemporáneos de la Facultad de Derecho y Ciencias Sociales de la Universidad de Belgrano. E-mail: fmilano@derecho.uba.ar
} 


\section{RÉSUMÉ}

L'objectif de la présente étude est de développer quelques idées autour des cultures juridiques comparées dans le cadre du Mercosur, particulièrement en ce qui concerne l'Argentine et le Brésil.

Mots-clés : Multiculturalisme ; Mercosur ; Brésil et Argentine.

Desde ya, dejamos planteada la necesidad de desarrollar esta temática en forma adecuada, atento los fenómenos de globalización-marginación de los tiempos actuales, que nos llevan a pensar en la idea de planetarización y posterior asimilación de los componentes culturales anglosajones, que en el caso en particular no se condicen con la realidad social de la Región ${ }^{2}$. Incluso en la actualidad, a partir del mencionado fenómeno, puede notarse la transformación del Derecho Comparado en Historia del Derecho ${ }^{3}$.

En los tiempos actuales denominados de la post-modernidad, es donde surge con fuerza la idea de ahondar el estudio de nuestras culturas para, a partir de allí, llegar a ciertas conclusiones que nos permitan pensar en aquellos elementos de originalidad que puedan extraerse de nuestros sistemas legales, y no pensemos en una mera recepción de otros sistemas foráneos, como ocurrió históricamente en América Latina, por ejemplo, con la influencia del Código Napoleón en el Derecho Civil o del Constitucionalismo norteamericano en el Derecho Constitucional ${ }^{4}$.

\footnotetext{
${ }^{2}$ Ciuro Caldani, Miguel Á. (2001). El Derecho Universal (Perspectiva para la ciencia jurídica de una nueva era), Rosario: Fundación para las Investigaciones Jurídicas; del mismo autor: (1993). "La tensión entre integración y dominación en el Derecho Universal de nuestro tiempo", en Revista del Centro de Investigaciones de Filosofía Jurídica y Filosofía Social, Rosario: FIJ, № 21, p. 64 y sigs.; también (1979). Lineamientos filosóficos del derecho universal, Rosario: Fundación para las Investigaciones Jurídicas.

${ }^{3}$ Puede verse Ciuro Caldani, Miguel Á. (1993). Bases Jusfilosóficas del Derecho de la Cultura, Rosario: Fundación para las Investigaciones Jurídicas; del mismo autor (2006). "Bases Culturales del Derecho Comparado", Rosario, en Revista del Centro de Investigaciones de Filosofía Jurídica y Filosofía Social, Rosario, FIJ, № 29, p. 113 a 138; también (2003). "Bases Culturales del Derecho Argentino", Rosario, en Revista del Centro de Investigaciones de Filosofía Jurídica y Filosofía Social, Rosario, FIJ, № 27, p. 113 a 126.; a su vez "Filosofía y Método del Derecho Comparado", en La Ley, t. 1989 C, p. 1080 y sigs.; también (1994). "La Actualidad, la Historia del Derecho y el Derecho Comparado", en Revista del Centro de Investigaciones de Filosofía Jurídica y Filosofía Social, Rosario, FIJ, № 23, p. 39 a 42. Acerca del Derecho Comparado en el orden internacional: David, René; Jauffret-Spinozi, Camille (2002). Les Grands Systèmes de Droit Contemporains, 11 a édition, Paris: Dalloz; Sweigert, Konrad, Kotz, Hein (2002). Introducción al Derecho Comparado, Colección Estudios Jurídicos, Oxford University Press; Losano, Mario (1982). Los grandes sistemas jurídicos, trad. Alfonso Ruiz Miguel, Madrid: Debate, p. 359 y ss.

${ }^{4}$ Puede verse Ciuro Caldani, Miguel Á. (1979). "Hacia una teoría general de la recepción del Derecho extranjero", en Revista de Direito Civil, 8, p. 73 y ss.; (1987). "Originalidad y recepción en el Derecho", en Boletín del Centro de Investigaciones de Filosofía Jurídica y Filosofía Social, Rosario, FIJ, № 9, p. 33 y ss.
} 
Se debería analizar en todo caso las diversas concepciones plasmadas en el Derecho Comparado para estudiar el fenómeno, como la idea de los trasplantes legales pensada por Watson; la de la recepción como fenómeno de sociología jurídica plasmada por Papachristos; la de la recepción como dinámica del Derecho Universal elaborada por Ciuro Caldani; o la idea de mestizaje, concepto utilizado por Sergue Gruzinski que hace referencia a un proceso de mezcla de ciertas tradiciones e instituciones del Derecho con otras, cerrando la puerta a un origen único ${ }^{5}$.

En nuestro caso en particular, es necesaria para el Mercosur la construcción de un marco ideal que permita respetar las diversas culturas a través de un análisis complejo, no limitando el mismo en forma simplista a través del juego excluyente de la Economía.

Para ello es dable valerse de una teoría jurídica en la integración del Mercosur, en este caso en particular haciendo especial referencia a la Argentina y al Brasil ${ }^{6}$.

\section{El abordaje histórico}

Es ineludible para afrontar la temática propuesta valernos de las lecciones de la Historia y sus relaciones con el Derecho ${ }^{7}$.

Pensamientos como los del gran jurista alemán Federico Carlos de Savigny plasmados en su inmortal "De la Vocación de Nuestro Siglo para la Legislación y la Ciencia del Derecho", son de excepcional enseñanza si pretendemos analizar las bases culturales del Derecho del Mercosur, si bien no debe ser interpretado en el sentido de una exacerbación de los nacionalismos atento la realidad social de Alemania en la época de elaboración de dicha obra. Resulta obvio que los fenómenos de integración precisamente

\footnotetext{
${ }^{5}$ Watson, Alan (1993). Legal transplants (an approach to comparative law), 2nd. Ed., Georgia: The University of Georgia Press; Papachristos, A.C. (1975). La réception des droits privés comme phénomène de sociologie juridique, Paris: Librairie Générale et de Jurisprudence; Ciuro Caldani (2003). Op. cit.; Gruzinski, Serge (1999). La pensée métisse, Paris: Librairie Arthéme Fayard; Meroi, Andrea A. (2006). "Marcos teóricos sobre el fenómeno de recepción jurídica", en Revista del Centro de Investigaciones de Filosofía Jurídica y Filosofía Social, Rosario, FIJ, № 29.

${ }^{6}$ Puede verse Ciuro Caldani, Miguel Á. (2000). "Papel de la teoría jurídica en la integración del Mercosur y del ámbito general de América del Sur", en Revista Investigación y Docencia, Rosario, FIJ, № 33, p. 65 a 84.

${ }^{7}$ Ciuro Caldani, Miguel Á. (1991/4). Lecciones de Historia de la Filosofía del Derecho, Rosario: Fundación para las Investigaciones Jurídicas.

${ }^{8}$ Savigny, Federico Carlos de (1977). De la vocación de nuestro siglo para la legislación y la ciencia del Derecho, Buenos Aires: Biblioteca Jurídica Heliasta (Los Grandes Maestros del Derecho) Vol. VII, Editorial Heliasta.
} 
apuntan a lo contrario, aunque no por ello debemos olvidar la noción de volkgeist o espíritu del pueblo, que puede ser traspolada a la de espíritu del pueblo mercosureño.

Si bien puede en la actualidad tildarse de "reduccionista" el análisis del Derecho en forma excluyente a través de la Historia, como fuera reflejado por la concepción tridimensional del Derecho de Werner Goldschmidt ${ }^{9}$, por lo demás complementación ejemplar entre Razón e Historia, no por ello se deben desconocer los aportes que puede brindar aquélla a las ciencias sociales en general y al Derecho en particular.

Podemos entonces razonar que las culturas hispánicas y portuguesas heredadas por la Argentina y el Brasil pueden en todo caso coexistir para, de producirse en forma exitosa un mestizaje, tal cual lo pensado por Gruzinski, podamos arribar a un sistema de derechos que presente ciertos rasgos de originalidad, valiéndose a su vez de aquellos aspectos valiosos que pueden haber sido o podrán ser recepcionados de otros sistemas.

Ciuro Caldani plantea la necesidad de observar las características más salientes de la cultura lusitana comparándola con la hispánica a través de la Historia, a los fines de razonar hasta qué punto su debido abordaje puede favorecer el avance del proceso de integración.

\section{a.-) El Brasil:}

Nos dice el mismo autor que Brasil heredó de la cultura lusitana la idea de desarrollo comercial a partir de la figura de Sebastiao José de Carvalho, Marqués de Pombal, quién dotó a Portugal en el siglo XVIII de una dinámica que pretendía acercarlo a la de los principales centros de poder en esos tiempos, en especial a Inglaterra ${ }^{10}$.

El ejemplo lo podemos observar en el intento de aumentar la producción nacional portuguesa con respecto a la competencia externa, de desarrollar el comercio colonial y de incentivar el desarrollo fabril.

Acorde con ello, en 1756 se fundó la Companhia para a Agricultura das vinhas do Alto Douro, a la que Pombal otorgó la exención de impuestos en las exportaciones y en el comercio con las colonias, estableciendo de ese modo la primer zona de producción

\footnotetext{
${ }^{9}$ Goldschmidt, Werner (1983). Introducción Filosófica al Derecho. La teoría trialista del mundo jurídico y sus horizontes. Buenos Aires: Editorial Depalma; Ciuro Caldani, Miguel Ángel (1976). Derecho y política, Bs. As: Depalma; del mismo autor (1982/84). Estudios de Filosofía Jurídica y Filosofía Política, Rosario: Fundación para las Investigaciones Jurídicas; también (1986). Estudios Jusfilosófícos, Rosario: Fundación para las Investigaciones Jurídicas.

${ }^{10}$ Ciuro Caldani, Miguel Á. "El marqués de Pombal, Portugal, Brasil y el Mercosur", en Derecho de la Integración, Rosario, FIJ, № 4, p. 113 y ss. Sobre la historia del Portugal puede verse Saraiva, José Hermano (1989). História de Portugal, Madrid: Alianza Editorial.
} 
vinícola demarcada en el mundo, colocando los famosos marcos pombalinos en los límites de la Región.

A la vez, el marqués estableció estímulos fiscales para la instalación de pequeñas empresas orientadas al mercado interno portugués, del que también formaban parte las colonias. Esa política proteccionista comprendía medidas que favorecían la importación de materias primas y encarecían los productos importados similares a los de fabricación portuguesa. Como resultado, surgieron en el país centenares de pequeñas industrias que producían los bienes más diversos.

Se pueden encontrar opiniones encontradas acerca de la injerencia del Marqués de Pombal en la política del Brasil, llegando incluso a ser tildado de tirano y opresor.

Un ejemplo de esta dicotomía lo podemos encontrar en materia de educación, con la violenta expulsión de los jesuitas en 1767, determinando que la educación en las colonias pasase a ser impartida por legos en las denominadas Aulas Regias.

O bien el intento de dar una mayor uniformidad cultural a la colonia, mediante la prohibición del Nheengatu, la lengua general (una mezcla de las lenguas nativas con el portugués, hablada por los bandeirantes) y haciendo obligatorio el uso del idioma portugués en todos los niveles. Algunos estudiosos de la historia afirman que debido a esta medida Brasil no llegó a ser un país bilingüe.

De cualquier manera, no puede negarse la influencia del Marqués de Pombal en el desarrollo de las políticas económicas en Portugal, y por herencia cultural, en el Brasil.

Quizás este dato histórico haya incidido de mejor manera en la recepción del Derecho Civil y Comercial francés en la codificación que se produjo en el siglo XIX en el Brasil, cuya obra cumbre fue el Esboço de Augusto Teixeira de Freitas, si lo comparamos con el mismo fenómeno producido en la Argentina a través de la obra de Dalmacio Vélez Sarsfield. Incluso el mismo Esboço influyó de manera decisiva en el Código Civil de la Argentina de 1871.

En la actualidad se encuentra en vigencia en Brasil el denominado Nuevo Código Civil, influenciado en forma directa por el pensamiento del filósofo paulista Miguel Reale, dado a luz ya en el siglo $X X I^{11}(10)$.

\footnotetext{
${ }^{11}$ Es posible ver Ley 10.406 del 20 de enero de 2002 ("Novo Código Civil Brasileiro", 2oed., prefacio del profesor Miguel Reale, San Pablo, Revista dos Tribunais, 2002; Martins-Costa, Judith; Branco, Gerson Luiz Carlos (2002). Diretrizes Teóricas do Novo Código Civil Brasileiro, San Pablo: Saraiva; Ciuro Cadani, Miguel
} 
Quizás la crítica que pueda hacerse a este cuerpo legal sea que haya llegado demasiado tarde a considerar los aspectos sociales del Derecho Civil, cuyo ejemplo paradigmático haya sido el Código Civil de Italia de 1942.

Pero si el hecho es analizado en forma más serena, se puede llegar a la conclusión de que los estamentos encargados de la elaboración de normas en el Brasil consideraron que, a lo largo de la segunda mitad del siglo XX, no estaban dadas las condiciones para la entrada en vigencia de un Código de las características que presenta en la actualidad.

Es de destacar la influencia de Reale en la consideración de la persona como valor fuente de todos los valores jurídicos, en opinión de Ciuro Caldani, prefiriendo el filósofo brasileño pensarla como ser humano más que como hombre ${ }^{12}$.

\section{b.-) La Argentina:}

En relación a la Argentina, la herencia de la cultura hispánica tradicional (considerada parasitaria) no permitió un desarrollo de una burguesía fuerte, e incluso cuando quiso ser llevada a cabo por influencia de Presidentes como Sarmiento o Mitre, fue notable el choque producido por las influencias de ciertos rasgos de la cultura angloafrancesada respecto a la hispánica tradicional más arriba mencionada, que llevaron a la desaparición del gaucho, ya que su perfil no encuadraba en las ideas liberales en pugna para la consolidación del Estado nacional ${ }^{13}$.

Es innegable que estas ideas imperantes hacia fines del siglo XIX y principios del siglo XX no pudieron concretarse atento la falta de una adecuado análisis de los componentes culturales.

Es por ello que el Código Civil argentino peca de un individualismo excesivo al abordar el derecho de la propiedad como un reflejo de la sociedad francesa de fines del siglo XIX, producto de la Revolución, cuando en la Argentina no existían en el momento de su entrada en vigencia las bases para poder implementarlo en forma exitosa.

Un dato fáctico de ese tiempo fue la denominada Conquista del Desierto, cuyo desenlace final data de 1879, a través de la cual se produjo un exterminio de los pueblos

\footnotetext{
Á. (2002). "Directrices iusfilósoficas del nuevo Código Civil brasileño", en Lexis Nexis - Jurisprudencia Argentina, 11 de diciembre de 2002, p. 13/19.

12 Puede verse Ciuro Caldani, Miguel Á. (2003). "Nuevas reflexiones sobre el Código Civil brasileño: los derechos de la persona y las uniones estables", en Investigación y Docencia, Rosario, FIJ, № 36, p. 9 a 13.

${ }^{13}$ Ciuro Caldani, Miguel Á. (1984). Op. cit.; del mismo autor Comprensión jusfilosófica del "Martín Fierro", Rosario: FIJ.
} 
originarios mapuche y tehuelche en el sur de la Argentina con el fin de poblar de habitantes civilizados esa Región y desarrollar las bases para la constitución del Estado nacional, que por lo demás no se encontraba en condiciones de ser llevada a cabo de la misma forma que en aquellos Estados europeos que, como Francia e Inglaterra, le sirvieron de parámetro.

Otro ejemplo de ese error de lectura de la realidad social lo representa la entrada en vigencia del Código Civil de 1871, fuertemente influenciado por la codificación francesa, haciendo culto del Derecho de la propiedad con un sentido decididamente liberal.

Si seguimos a Savigny, ya en sus tiempos notaba las improvisaciones efectuadas en la elaboración del propio Code Napoleon, ejemplificando acerca de la consideración del Derecho romano como fuente en forma inexacta ${ }^{14}$.

Por ejemplo, la ácida crítica realizada a Bigot de Preameneu, a quién lo tilda de superficial en sus razonamientos. O a Malville, a quién según "sus escritos se lo muestra como hombre honorable e inteligente, pero carente de erudición jurídica”, para luego sostener acerca de él como así también de Portalis que

“(...) parten de una historia del divorcio romano, no sólo errónea, sino imposible. Así, a modo de ejemplo, opinan que el matrimonio no podía entre los romanos ser disuelto por la voluntad de uno sólo de los cónyuges, sino que se requería el mutuo consentimiento de ambos, con lo que todo cuanto ha sido dicho por Justiniano acerca de esta materia no tiene sentido alguno, y el divorcio por convención entre los romanos es una simple consecuencia de la falsa idea, según la que el matrimonio se debía considerar en la misma línea que todos los demás contratos $(. . .)^{15 ”}$.

A este hecho apuntado por Savigny se le suman las citas que en el articulado del Código Civil argentino efectúa Dalmacio Vélez Sarsfield mencionando en forma expresa a los juristas que redactaron la obra francesa, como Pothier (por lo demás, el menos vilipendiado por Savigny), o incluso a los comentaristas del mismo cuerpo legal, como Demolombe, Zachariae u Ortolan, para dar algunos ejemplos, quiénes a su vez basaron sus citas en el Derecho romano.

\footnotetext{
${ }_{15}^{14}$ Savigny (1977).Op. cit.

${ }^{15}$ Ibidem, p. 90-92.
} 
Sin duda, lo que sí ha sido importante por parte de los juristas de Argentina fue visualizar la necesidad de reformar el Código en el año 1968, dotando al cuerpo de un contenido social que hasta el momento no reconocía, sin dudas inspirada dicha reforma en el Código Civil de Italia de 1942.

Podríamos mencionar al artículo 1198 en su párrafo primero, que establece el principio de la buena fe en lo que refiere a la celebración, interpretación y ejecución de los contratos, o lo dispuesto en el segundo párrafo del mismo artículo, que esboza la teoría de la imprevisión.

O bien lo expresado por el artículo 2513, que establece el ejercicio regular de los derechos reales.

Quizás estos logros pudieron haberse obtenido con anterioridad si el desarrollo del movimiento peronista, que representó a las masas populares a partir de la década del 40, no se hubiera visto truncado por diversos golpes de estado, que permitieron el arribo al gobierno nacional de las fuerzas armadas, aunque esta es una tarea en todo caso conjetural $^{16}$.

Incluso siguiendo la línea de la Historia, el Brasil pretendía lo mismo por influjo del gobierno de Getúlio Vargas.

Si analizamos por ejemplo al Derecho Constitucional, el gobierno de Perón intentó reformar la Constitución Nacional argentina en el año 1949, hecho que en definitiva no prosperó, y Vargas lo hizo con anterioridad en 1934, lo que le permitió asumir el poder en el Brasil, siendo ambas Cartas Fundamentales notoriamente influenciadas por la Constitución alemana de Weimar de 1919, que junto a la Constitución de Méjico de 1917 dieron origen al Constitucionalismo social en el mundo.

Esto demuestra también que en toda sociedad deben tenerse en cuenta las condiciones socio-políticas para poder implementar una adecuada recepción de los elementos de otros sistemas jurídicos para que los mismos puedan ser asimilados y no directamente rechazados ${ }^{17}$.

\footnotetext{
${ }^{16}$ Sobre el particular puede verse Romero, José Luis (1982). Las ideologías de la cultura nacional y otros ensayos, Buenos Aires: Centro Editor de América Latina; también Feinman, José Pablo (2010). El Peronismo. Filosofía política de una persistencia argentina, Buenos Aires: Editorial Planeta, Tomo I.

${ }^{17}$ Puede verse Ciro Caldani, Miguel Á. (2003). Op. cit.
} 


\section{La realidad del MERCOSUR}

Las características actuales del proceso de integración mercosureño permiten pensar en que los fenómenos de recepción de otros sistemas jurídicos pueden prosperar, si se toman debidamente en cuenta los datos históricos y de la realidad social existentes en la Región.

Podemos arribar a la conclusión de que en la misma tuvo primacía la lengua tupíguaraní, propia de los pueblos originarios, y que reinaba en un territorio tan amplio que abarcaba parte de los actuales de Argentina, Bolivia, Brasil, Paraguay, e incluso de lugares mucho más alejados como Colombia, Guayana Francesa, Perú y Venezuela.

Por lo demás, aún luego de los procesos de colonización, las lenguas imperantes quedaron solamente reducidas a dos, el castellano y el portugués, las cuales son lenguas derivadas del latín, con muchos más puntos de coincidencias que de divergencias.

Para dar un ejemplo en contrario, Europa hoy convive en una Unión de sus pueblos mediante la existencia de veinte lenguas oficiales, que podrían ampliarse de sumarse Croacia o Turquía al proceso de integración, por nombrar a dos de los Estados que han solicitado su incorporación como miembros plenos.

Incluso desde el punto de vista geográfico, las fronteras nacionales han establecido límites políticos allí dónde parecería existir una unidad, como por ejemplo, en la región de la triple frontera, la zona del Chaco o la de la Mesopotamia, que podría extenderse esta última sin ningún tipo de dificultad al territorio del Uruguay. En realidad, sería más correcto analizar la geografía de la Región desde un punto de vista geológico ante la existencia del Acuífero Guaraní.

A su vez, se puede arribar a la conclusión de que no existieron importantes conflictos bélicos, con excepción de la Guerra de la Triple Alianza, que hayan obstaculizado los intentos de convivencia pacífica de las culturas imperantes en esta parte del mundo, como sí ha ocurrido en forma brutal en la civilizada Europa, que fue víctima de los dos grandes conflictos mundiales.

También podemos arribar a la conclusión que desde el punto de vista de la Religión, en nuestra Región podemos encontrar elementos coincidentes a través del catolicismo heredado de España y Portugal, debiendo también respetarse las múltiples creencias de los pueblos originarios. 
No ocurre lo mismo en Europa, en dónde la intolerancia religiosa en más de una oportunidad motivó conflictos bélicos, ante la coexistencia, muchas veces conflictiva, de católicos apostólicos romanos, católicos apostólicos ortodoxos y protestantes, por nombrar a los credos más representativos. Incluso la influencia del protestantismo (en especial, dentro de este último, el calvinismo) en el desarrollo posterior del capitalismo es evidente ${ }^{18}$.

Desde el punto de vista del Derecho, siguiendo con el análisis comparativo entre el Mercosur y la Unión Europea, en esta última deben convivir los sistemas del Derecho Continental y del Common Law, junto con algunos aspectos, por ejemplo, del Derecho Ruso, que en la era soviética influyó en forma considerable en muchos Estados del Centro y del Este de Europa, como ser los de Bulgaria, Hungría, Polonia, o los países bálticos, por nombrar sólo a algunos.

Si en este viaje imaginario nos trasladamos al Mercosur, podemos observar que en todo caso fue sólo uno el sistema que influyó en forma decisiva, el Romano-Germánico o Continental Europeo, que por las peculiares características que fue adoptando, ameritó comenzar a denominarse sub-sistema ${ }^{19}$.

Incluso en el ámbito del Derecho Privado, la influencia del Esboço de Augusto Teixeira de Freitas en el Código Civil argentino fue notable.

Es por todas estas razones que podemos arribar a la conclusión que pensar en un Derecho Privado común para los Estados que conforman el Mercosur no nos parece irrealizable, si por supuesto se toman debidamente en cuenta las enseñanzas del estudio de la Historia y de la realidad social imperante ${ }^{20}$.

El Mercosur cuenta con elementos que permiten pensar en este fenómeno, y ello llevó a la entrada en vigencia del Protocolo de Buenos Aires sobre Jurisdicción en Materia Contractual, que por ejemplo, establece la posibilidad de prórroga de jurisdicción si la misma es acordada en forma expresa por las partes, tomando en cuenta un acuerdo de

\footnotetext{
${ }^{18}$ Weber, Max (1973). La ética protestante y el espíritu del capitalismo, traducción de Luis Legaz y Lacambra, 2 $2^{\mathrm{a}}$. Edición, Barcelona: Península.

${ }^{19}$ Puede verse (2008). "Roma y América. Diritto Romano Comune." (Director Schipani, Sandro), en Revista de Derecho de la Integración y Unificación del Derecho en Europa y América Latina, Bogotá, publicación de la Universidad Externado de Colombia.

20 Dreyzin de Klor, Adriana S. (1997). EI MERCOSUR, Generador de una nueva fuente de Derecho Internacional Privado, Buenos Aires: Zavalía Editor.
} 
elección del foro a favor de un Juez de un Estado parte, y exista una conexión razonable según las normas de jurisdicción del cuerpo normativo ${ }^{21}$.

Pero entiendo que aún se puede avanzar mucho más en la profundización de aspectos uniformadores y unificadores, del Derecho Privado de fondo, tomando como ejemplo los principios del Instituto Internacional para la Unificación del Derecho Privado (UNIDROIT) en materia preferentemente mercantil, unificando criterios en base a los siguientes temas: formación de los contratos; interpretación de los contratos; requisitos de validez; cumplimiento e incumplimiento de los contratos; daños y perjuicios por incumplimiento; enriquecimiento injusto o indebido y restitución; y pruebas.

Ya Savigny indicaba que la convergencia hacia la profundización de la comunidad jurídica de los Estados, como consecuencia de la coincidencia de los criterios de conexión conllevaría a la construcción de normas de conflicto uniformes a escala internacional y consecuentemente a la armonía de las decisiones en el ámbito internacional ${ }^{22}$.

Trayendo este pensamiento a los tiempos actuales, bien podría aplicarse este criterio en la búsqueda de normas de armonización en el ámbito de la integración regional.

Aún así se puede avanzar en el Mercosur en el tratamiento de temas más complejos de abordar en forma uniformadora o unificadora, como ciertos aspectos del Derecho de Familia, que hacen a la consideración del matrimonio, la filiación, la adopción, el divorcio vincular; o nuevas temáticas relacionadas a las familias recompuestas, monoparentales o las relacionadas a las uniones matrimoniales entre personas del mismo sexo, de por sí puntos conflictivos de abordar en todo sentido ${ }^{23}$.

De cualquier manera, la riqueza del análisis debe basarse en el respeto de las distintas individualidades y ver de qué manera las mismas pueden convivir en forma armonizada.

Evidentemente si se pretende llevar a cabo esta monumental tarea con cierto éxito, deben estudiarse en principio criterios armonizadores, tratando de respetar las diversas

\footnotetext{
${ }^{21}$ Puede verse Deyzin de Klor, Adriana S. (1996). "Jurisdicción internacional contractual en el Mercosur", en Revista de Derecho Privado y Comunitario, Santa Fe: Rubinzal-Culzoni Editores, № 7, p. 465 a 491.

${ }_{22}$ Sobre el particular puede verse David, René. "The methods of unification", en http://www.cisg.law.pace.edu/cisg/biblio/david.html

${ }^{23}$ Puede verse Feldstein de Cárdenas, Sara L. "El conflicto de multiculturalidad ¿Réquiem para el orden público internacional en materia de familia?", en http://www.eldial.com/nuevo'; también Ciuro Caldani, Miguel Á. "Visión sintética del Derecho Comparado desde el punto de vista cultural, con especial referencia al Derecho de Familia", en Investigación y Docencia, Rosario, FIJ, № 30, p. 95 sigs.
} 
naturalezas de estas instituciones del Derecho de la Familia, para una vez iniciada la consolidación de ciertas cuestiones, pretender de ser posible ello arribar a una unificación del Derecho.

Evidentemente partimos de la base de considerar a la familia como una institución cultural, y no natural.

En este aspecto, es de destacar el accionar actual del Tribunal Superior de Justicia del Brasil, que con tolerancia cultural y personalización para proteger los intereses de los inmigrantes y descendientes japoneses en Brasil, hizo respetar las diferencias culturales entre ambos países y fomentar la armonía entre Brasil y Japón.-

A su vez, en la Argentina la línea de tensión en este sentido se presenta en la convivencia entre los pueblos originarios y la inmigración procedente de países europeos como España e Italia en mayor medida, cuyas calificaciones acerca del concepto de familia son muy disímiles entre sí, lo que exige un esfuerzo en el hecho de comprender y tolerar el pensamiento del otro.

\section{A manera de conclusión}

Se puede arribar a la conclusión de que es válido en la realidad del proceso de integración mercosureño asumir la existencia de sus diversas culturas jurídicas, ya que a partir de allí puede afrontarse de mejor manera los efectos de la presente era de la postmodernidad, que de por sí genera confusión, cuando no perplejidad.

Vale entonces remarcar el valor de algunas conclusiones que me parecen muy esclarecedoras: "La complejidad del mundo hace que el orden jurídico y sus categorías fundantes sean cada vez más inaprensibles. Pensar el derecho para la multiculturalidad, que ya no puede ser negada, importa revisar las piezas centrales del montaje jurídico", porque "conmueve la proximidad de lo distinto, de lo que no es igual, del otro y de los otros, cuya presencia no es transitoria y fugaz, sino que se instala entre nosotros y nos interroga acerca de quiénes somos"24.

\footnotetext{
${ }^{24}$ Ruiz, Alicia E. C. (2003). "El Derecho como discurso y como Juego". Revista Jurídica Universidad Interamericana de Puerto Rico, Septiembre-diciembre.
} 


\section{Referencias Bibliográficas}

Ciuro Caldani, Miguel Á. (2001). El Derecho Universal (Perspectiva para la ciencia jurídica de una nueva era), Rosario: Fundación para las Investigaciones Jurídicas.

Ciuro Caldani, Miguel Á. (1993). "La tensión entre integración y dominación en el Derecho Universal de nuestro tiempo", en Revista del Centro de Investigaciones de Filosofía Jurídica y Filosofía Social, Rosario: FIJ, № 21.

Ciuro Caldani, Miguel Á. (1979). Lineamientos filosóficos del derecho universal, Rosario: Fundación para las Investigaciones Jurídicas.

Ciuro Caldani, Miguel Á. (1993). Bases Jusfilosóficas del Derecho de la Cultura, Rosario: Fundación para las Investigaciones Jurídicas.

Ciuro Caldani, Miguel Á. (2006). "Bases Culturales del Derecho Comparado", Rosario, en Revista del Centro de Investigaciones de Filosofía Jurídica y Filosofía Social, Rosario, FIJ, № 29.

Ciuro Caldani, Miguel Á. (2003). "Bases Culturales del Derecho Argentino", Rosario, en Revista del Centro de Investigaciones de Filosofía Jurídica y Filosofía Social, Rosario, FIJ, № 27.

Ciuro Caldani, Miguel Á. "Filosofía y Método del Derecho Comparado", en La Ley, t. 1989 C.

Ciuro Caldani, Miguel Á. (1994). "La Actualidad, la Historia del Derecho y el Derecho Comparado", en Revista del Centro de Investigaciones de Filosofía Jurídica y Filosofía Social, Rosario, FIJ, № 23.

Ciuro Caldani, Miguel Á. (1976). Derecho y política, Buenos Aires: Depalma.

Ciuro Caldani, Miguel Á. (1982/84). Estudios de Filosofía Jurídica y Filosofía Política, Rosario: Fundación para las Investigaciones Jurídicas.

Ciuro Caldani, Miguel Á. (1986). Estudios Jusfilosófícos, Rosario: Fundación para las Investigaciones Jurídicas.

Ciuro Caldani, Miguel Á. "El marqués de Pombal, Portugal, Brasil y el Mercosur", en Derecho de la Integración, Rosario, FIJ, № 4.

Ciuro Caldani, Miguel Á. (2000). "Papel de la teoría jurídica en la integración del Mercosur y del ámbito general de América del Sur", en Revista Investigación y Docencia, Rosario, FIJ, № 33.

Ciuro Caldani, Miguel Á. (1991/4). Lecciones de Historia de la Filosofía del Derecho, Rosario: Fundación para las Investigaciones Jurídicas. 
Ciuro Caldani, Miguel Á. (1979). "Hacia una teoría general de la recepción del Derecho extranjero", en Revista de Direito Civil, 8.

Ciuro Caldani, Miguel Á. (1987). "Originalidad y recepción en el Derecho", en Boletín del Centro de Investigaciones de Filosofía Jurídica y Filosofía Social, Rosario, FIJ, № 9.

Ciuro Caldani, Miguel Á. (2002). "Directrices iusfilósoficas del nuevo Código Civil brasileño", en Lexis Nexis - Jurisprudencia Argentina, 11 de diciembre de 2002.

Ciuro Caldani, Miguel Á. (2003). "Nuevas reflexiones sobre el Código Civil brasileño: los derechos de la persona y las uniones estables", en Investigación y Docencia, Rosario, FIJ, № 36 .

Ciuro Caldani, Miguel Á.. Comprensión jusfilosófica del "Martín Fierro”, Rosario: FIJ.

Ciuro Caldani, Miguel Á. "Visión sintética del Derecho Comparado desde el punto de vista cultural, con especial referencia al Derecho de Familia", en Investigación y Docencia, Rosario, FIJ, № 30 .

David, René; Jauffret-Spinozi, Camille (2002). Les Grands Systèmes de Droit Contemporains, $11^{\text {a }}$ édition, Paris: Dalloz.

David, René. "The methods of unification", en

http://www.cisg.law.pace.edu/cisg/biblio/david.html

Dreyzin de Klor, Adriana S. (1997). El MERCOSUR, Generador de una nueva fuente de Derecho Internacional Privado, Buenos Aires: Zavalía Editor.

Dreyzin de Klor, Adriana S. (1996). "Jurisdicción internacional contractual en el Mercosur", en Revista de

Derecho Privado y Comunitario, Santa Fe: Rubinzal-Culzoni Editores, № 7.

Feinman, José Pablo (2010). El Peronismo. Filosofía política de una persistencia argentina, Buenos Aires: Editorial Planeta, Tomo I.

Feldstein de Cárdenas, Sara L. "El conflicto de multiculturalidad ¿Réquiem para el orden público internacional en materia de familia?", en http://www.eldial.com/nuevo

Goldschmidt, Werner (1983). Introducción Filosófica al Derecho. La teoría trialista del mundo jurídico y sus horizontes. Buenos Aires: Editorial Depalma.

Gruzinski, Serge (1999). La pensée métisse, Paris: Librairie Arthéme Fayard.

Losano, Mario (1982). Los grandes sistemas jurídicos, trad. Alfonso Ruiz Miguel, Madrid: Debate. 
Martins-Costa, Judith; Branco, Gerson Luiz Carlos (2002). Diretrizes Teóricas do Novo Código Civil Brasileiro, San Pablo: Saraiva.

Meroi, Andrea A. (2006). "Marcos teóricos sobre el fenómeno de recepción jurídica", en Revista del Centro de Investigaciones de Filosofía Jurídica y Filosofía Social, Rosario, FIJ, № 29.

Papachristos, A.C. (1975). La réception des droits privés comme phénomène de sociologie juridique, Paris: Librairie Générale et de Jurisprudence.

Romero, José Luis (1982). Las ideologías de la cultura nacional y otros ensayos, Buenos Aires: Centro Editor de América Latina.

Ruiz, Alicia E. C. (2003). "El Derecho como discurso y como Juego". Revista Jurídica Universidad Interamericana de Puerto Rico, Septiembre-diciembre.

Saraiva, José Hermano (1989). História de Portugal, Madrid: Alianza Editorial.

Savigny, Federico Carlos de (1977). De la vocación de nuestro siglo para la legislación y la ciencia del Derecho, Buenos Aires: Biblioteca Jurídica Heliasta (Los Grandes Maestros del Derecho) Vol. VII, Editorial Heliasta.

Sweigert, Konrad; Kotz, Hein (2002). Introducción al Derecho Comparado, Colección Estudios Jurídicos, Oxford University Press.

Watson, Alan (1993). Legal transplants (an approach to comparative law), 2nd. Ed., Georgia: The University of Georgia Press;

Weber, Max (1973). La ética protestante y el espíritu del capitalismo, traducción de Luis Legaz y Lacambra, 2ª . Edición, Barcelona: Península.

Recebido para publicação em junho de 2011.

Aprovado para publicação em dezembro de 2011. 